\title{
On the Khovanov Homology of 2- and 3-Strand Braid Links
}

\author{
Abdul Rauf Nizami1, Mobeen Munir1', Tanweer Sohail'2, Ammara Usman' \\ ${ }^{1}$ Division of Science and Technology, University of Education, Lahore, Pakistan \\ ${ }^{2}$ University of Science and Technology of China, Hefei, China \\ Email: arnizami@ue.edu.pk,mmunir@ue.edu.pk, tsohail@mail.ustc.edu.cn, ammarausman88@ymail.com
}

Received 19 January 2016; accepted 28 May 2016; published 31 May 2016

Copyright (C) 2016 by authors and Scientific Research Publishing Inc.

This work is licensed under the Creative Commons Attribution International License (CC BY).

http://creativecommons.org/licenses/by/4.0/

c) (i) Open Access

\section{Abstract}

Although computing the Khovanov homology of links is common in literature, no general formulae have been given for all of them. We give the graded Euler characteristic and the Khovanov homology of the 2-strand braid link $\widehat{x_{1}^{n}}, n \geq 2$, and the 3 -strand braid $\alpha(n)=x_{1} x_{2} x_{1} x_{2} \cdots$.

\section{Keywords}

Khovanov Homology, Khovanov Bracket, Graded Euler Characteristic, Braid Link, Jones Polynomial

\section{Introduction}

Khovanov homology is an invariant for oriented links which was introduced by Mikhail Khovanov in 2000 as a categorification of the Jones polynomial [1].

Khovanov assigned a bigraded chain complex $C^{i, j}(L)$ to the oriented link diagram $L$ whose differential was graded of bidegree $(1,0)$ and whose homotopy type depended only on the isotopy class of $L$. The bigraded homology group $H^{i, j}(D)$ of the chain complex $C^{i, j}(D)$ provides an invariant of oriented links, now known as Khovanov homology.

Although Khovanov's construction is combinatorial from which Khovanov homology is algorithmically computable, we shall follow rather a simple way of Bar-Natan's, which he introduced in [2] to compute the Khovanov homology.

\section{Links and Link Invariants}

A link in $\mathbb{R}^{3}$ is a finite collection of disjoint circles smoothly embedded in $\mathbb{R}^{3}$. These circles are called the 
components of the link. If an orientation of the components is specified, we say that the link is oriented. A link consisting of only one component is called a knot.

Links are usually studied via projecting them on the plane. A projection with information of over- and undercrossing is called a link diagram. Some link diagrams are given in Figure 1.

Two links are called isotopic (or equivalent) if one of them can be transformed to another by a diffeomorphism of the ambient space $\mathbb{R}^{3}$ onto itself. Two isotopic knots are given in Figure 2.

Remark 1. By a link we shall mean a diagram of its isotopy class.

Reidemeister gave in [3] a fundamental result about the equivalence of two links: Two Links are equivalent if and only if one can be transformed into the other by a finite sequence of ambient isotopies of the plane and the local Reidemeister moves given in Figure 3.

To classify links one needs a link invariant [4], a functions I: Links $\rightarrow$ \{numbers or polynomials or colours, etc.\} that gives one value for all links in an isotopy class of links and gives different values, but not always, for different classes of links. To check whether a function is a link invariant one has to show that it is invariant under all the Reidemeister moves. This paper is concerned with the link invariants: the Khovanov homology and the Jones polynomial.

\section{Braids}

An $n$-strand braid is a set of $n$ non-intersecting smooth paths connecting $n$ points on a horizontal plane to $n$ points exactly below them on another horizontal plane in an arbitrary order [5]. The smooth paths are called strands of the braid. A 2-strand braid is given in Figure 4.

The product $a b$ of two $n$-strand braids is defined by putting the braid $a$ above the braid $b$ and then gluing their common end points. A braid with only one crossing is called elementary braid. The ith elementary braid $x_{i}$ on $n$ strands is given in Figure 5.

A useful property of elementary braids is that every braid can be written as a product of elementary braids. For instance, the above 2-strand braid is $x_{i}^{-3}=\left(x_{i}^{-1}\right)\left(x_{i}^{-1}\right)\left(x_{i}^{-1}\right)$.

The closure of a braid $b$ is the link $\hat{b}$ obtained by connecting the lower ends of $b$ with the corresponding upper ends, as you can see in Figure 6. An important result by Alexander connecting knots and braids is:

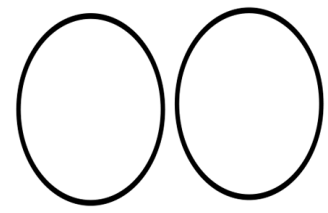

Trivial 2-component link

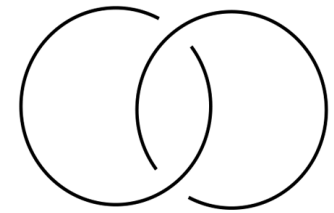

Hopf link

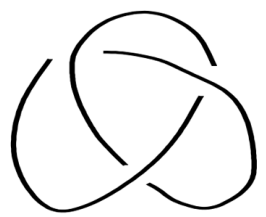

Trefoil knot

Figure 1. Link diagrams.
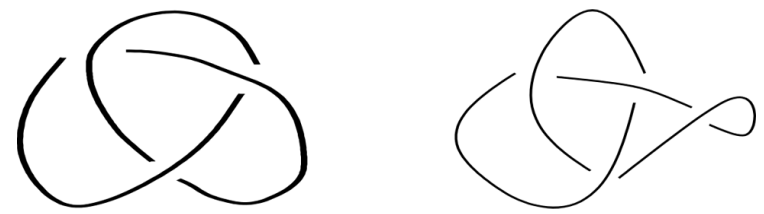

Figure 2. Isotopic knots.

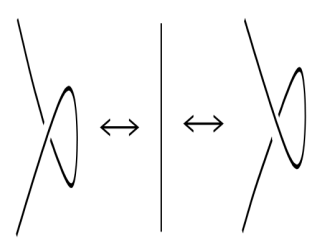

$R_{1}$

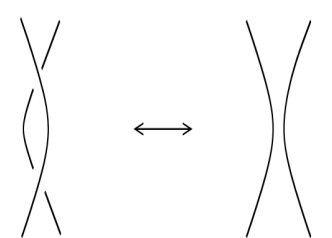

$R_{2}$

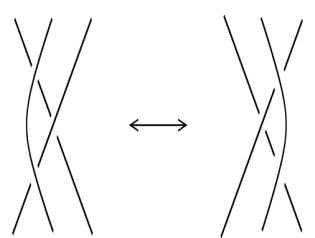

$R_{3}$

Figure 3. Reidemeister moves. 


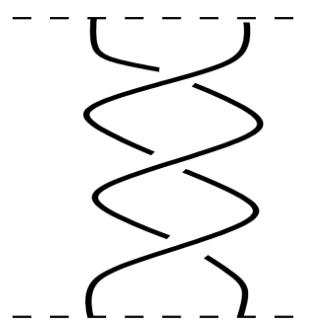

Figure 4. A 2-strand braid.

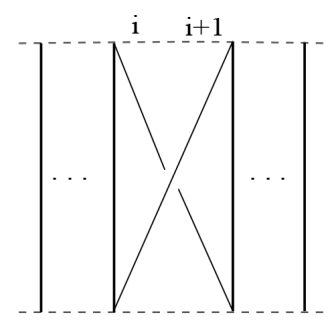

Figure 5. Elementary braid $x_{i}$.

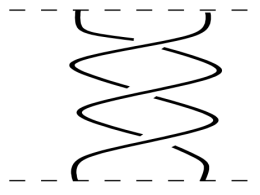

$b$

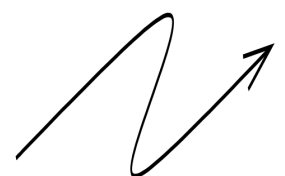

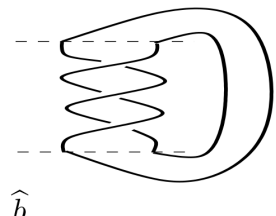

Figure 6. Closure of a braid.

Theorem 1. [6] Each link can be represented as the closure of a braid.

\section{The Kauffman Bracket and the Jones Polynomial}

In 1985 V. F. R. Jones revolutionized knot theory by defining the Jones polynomial as a knot invariant via Von Neumann algebras [7]. However, in 1987 L. H. Kauffman introduced a state-sum model construction of the Jones polynomial that was purely combinatorial and remarkably simple [8].

A Kauffman state $s$ of a link $L$ is obtained by replacing each crossing $(メ)$ of $L$ with the 0 -smoothing $〉$ or the 1-smoothing $\asymp$ (so that the result is a disjoint union of circles embedded in the plane). We denote by $\mathcal{S}(L)$ the set of all Kauffman states of $L$. A smoothing of trefoil knot is given in Figure 7 .

Let $s$ be a state in $\mathcal{S}(L), \gamma(s)$ the number of circles in the state, and $\alpha(s)$ and $\beta(s)$ the numbers of crossings in states 0 and 1 . Then the Kauffman bracket for $L$ is defined by the relation

$$
\langle L\rangle=\sum_{s} q^{\alpha(s)-\beta(s)}\left(-q^{2}-q^{-2}\right)^{\gamma(s)-1} .
$$

It is well known that the Kauffman bracket satisfies the relations:

$$
\begin{gathered}
\langle L\rangle=q\left\langle L_{0}\right\rangle+q^{-1}\left\langle L_{1}\right\rangle \\
\langle L \sqcup \bigcirc\rangle=\left(-q^{2}-q^{-2}\right)\langle L\rangle \\
\langle\bigcirc\rangle=1
\end{gathered}
$$

This bracket is not invariant under the first Reidemeister move [9], see, for instance, [4]. To overcome this difficulty, one needs something more: Let us consider that the link diagram $L$ is now oriented. Then each crossing appears either as $X$, which is called the positive crossing or as $X$, which is called the negative crossing. If we denote the number of positive crossings by $n_{+}$and the number of negative crossings by $n_{-}$, then the unnormalized Jones polynomial is defined by the relation 


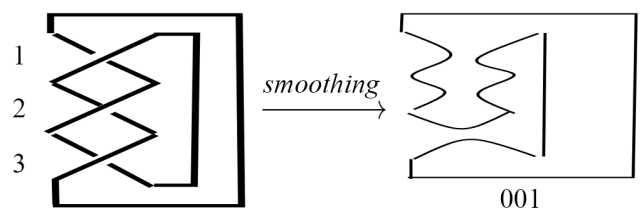

Figure 7. 0- and 1-smoothings.

$$
\hat{J}(L)=(-1)^{n_{-}} q^{n_{+}-2 n_{-}}\langle L\rangle
$$

and its normalized version by the relation

$$
J(L)=\frac{1}{q+q^{-1}} \hat{J}(L) .
$$

Since this polynomial is invariant under all three Reidemeister moves, it is an invariant for oriented links.

Example 1. It is easy to check that the normalized Jones polynomial of the link $\widehat{x_{1}^{3}}$ : $J\left(\widehat{x_{1}^{3}}\right)=-q^{5}+q^{3}+q^{-1}$

\section{On the Way to Khovanov Homology}

Definition 1. A graded vector space $W$ is a decomposition of $W$ into a direct sum of the form

$$
W=\oplus_{m \in I} W_{m},
$$

where each $\left\{W_{m}\right\}$ is a homogeneous component with degree $m$ of the graded vector space $W$.

Definition 2. Let $V$ and $W$ be two homogeneous components of graded vector spaces. The degree of the tensor product $V \otimes W$ is the sum of the degrees of $V$ and $W$.

Definition 3. Let $W=\oplus_{m} W_{m}$ be a graded vector space with homogeneous components $\left\{W_{m}\right\}$. The graded dimension of $W$ is the power series

$$
q \operatorname{dim} W:=\sum_{m} q^{m} \operatorname{dim} W_{m} .
$$

Definition 4. The degree shift.$\{l\}$ of a graded vector space $W=\oplus W_{m}$ is defined by $(W .\{l\})_{m}:=W_{m-l}$, so that $q \operatorname{dim} W .\{l\}=q^{l}$ qdimW .

Definition 5. Bar-Natan discovered in [2] that Khovanov's idea was to replace the Kauffman bracket what he called the Khovanov bracket $\llbracket L \rrbracket$, which is a chain complexample of graded vector spaces whose graded Euler characteristic is $\langle L\rangle$. Likewise the Kauffman bracket, the Khovanov bracket is defined by the axioms:

$$
\llbracket L \rrbracket=0 \rightarrow \mathbb{Z} \rightarrow 0, \llbracket L \sqcup \bigcirc \rrbracket=V \otimes \llbracket L \rrbracket,
$$

and

$$
\text { toremovenumbering (beforeeachequation) } \llbracket \backslash \rrbracket=0 \rightarrow \llbracket\rangle\langle\rrbracket \stackrel{d}{\longrightarrow} \longleftarrow \llbracket \llbracket\{1\} \rightarrow 0 .
$$

Here $V$ is a graded vector space with graded dimension $q+q^{-1}$.

Definition 6. The chain complexample $\bar{C}$ of graded vector spaces $\bar{C}^{r}$ (where the grading $r$ is the "height" of a piece $\bar{C}^{r}$ of that complexample) is defined as:

$$
\cdots \longrightarrow \bar{C}^{r-1} \stackrel{d^{r-1}}{\longrightarrow} \bar{C}^{r} \stackrel{d^{r}}{\longrightarrow} \bar{C}^{r+1} \stackrel{d^{r+1}}{\longrightarrow} \cdots
$$

The height shift operation . $[s]$ on the chain complexample $\bar{C}$ is defined: if $C=\bar{C}[s]$, then $C^{r}=\bar{C}^{r-s}$.

Definition 7. The graded Euler characteristics of a chain complexample is defined to be the alternating sum of the graded dimensions of its homology groups, i.e.

$$
\chi_{q}(C):=\sum_{r}(-1)^{r} q \operatorname{dim} H^{r} .
$$


Theorem 2. [2] If the degree of the differential is zero and if all the chain groups are finite dimensional, $\chi_{q}(C)$ is also equal to the alternating sum of the graded dimensions of the chain groups, i.e.

$$
\chi_{q}(C):=\sum_{r}(-1)^{r} q \operatorname{dim} C^{r} .
$$

Theorem 3. [2] The graded Euler characteristic of $C(L)$ is equal to the unnormalized Jones polynomial of L, i.e. $\chi_{q}(C(L))=\hat{J}(L)$.

Now we give the graded Euler characteristic of $\widehat{x_{1}^{3}}$. First, some terminology: By the symbols $L, \mathcal{X}, n, n_{+}$, and $n_{-}$we shall mean the oriented link diagram, the set of crossings in $L$, the number of crossings in $L$, the number of positive crossings and the number of negative crossings in $L$, respectively. Let $V$ be the graded vector space with two basis elements $v_{ \pm}$whose degrees are \pm 1 respectively, so that $q \operatorname{dim} V=q+q^{-1}$. With every vertexample $\alpha$ of the cube $\{0,1\}^{\mathcal{X}}$ we associate the graded vector space $V_{\alpha}(L):=V^{\otimes k}\{r\}$, where $k$ is the number of cycles in the smoothing of $L$ corresponding to $\alpha$ and $r$ is the height $|\alpha|=\Sigma_{i} \alpha_{i}$ of $\alpha$. We then set the $r$ th chain group $\llbracket L \rrbracket^{r}$ (for $0 \leq r \leq n$ ) to be the direct sum of all the vector spaces at height $r: \llbracket L \rrbracket^{r}:=\oplus_{\alpha: r=|\alpha|} V_{\alpha}(L)$.

Before computing the Khovanov homology, we define two gradings, the homological grading and the quantum grading. The homological grading of the chain complexample is defined as $\operatorname{gr}(x)=c_{1}(v)-n_{-}$, where $x \in C(L)$ and $c_{1}(v)$ is the number of 1-smoothings in the coordinates of V. In case of chain complexample, the quantum grading of the chain groups is $q(x)=-p(x)+\operatorname{gr}(x)+n_{+}-n_{-}$and is

$q(x)=p(x)+\operatorname{gr}(x)+n_{+}-n_{-}$in case of co-chain complexample. Now onward we shall use the notation $K h^{r, q}$ for the Khovanov homology, where the first inexample $r$ indicates the homological grading and the second indexample $q$ indicates the quantum grading. We need these gradings to compute the Jones polynomial from the Khovanov homology.

Example 2. Here is the Khovanov homology of $\widehat{x_{1}^{3}}$ :

1) The n-cube: The 3-cube of the trefoil knot $\widehat{x_{1}^{3}}$ is given in Figure 8.

2) Khovanov Bracket: The Khovanov brackets along with their q-dimensions are given in Table 1.

3) Unnormalized Jones polynomial: The graded Euler characteristic of $\widehat{x_{1}^{3}}$ is

$$
\chi_{q}\left(\widehat{x_{1}^{3}}\right)=\left(q+q^{-1}\right)^{2}-3 q\left(q+q^{-1}\right)+3 q^{2}\left(q+q^{-1}\right)^{2}-q^{3}\left(q+q^{-1}\right)^{3}=q^{-2}+1+q^{2}-q^{6} .
$$

4) Khovanov Homology: In order to compute the Khovanov homology of $\widehat{x_{1}^{3}}$, we multiply the unnormalized Jones polynomial with the factor $\left[(-1)^{n_{-}} q^{n_{+}-2 n_{-}}\right]$, where in our case is $\left(n_{-}, n_{+}\right)=(0,3)$.

$$
\hat{J}\left(\widehat{x_{1}^{3}}\right) q^{3}=(-1)^{3} q^{9}+q^{5}+q^{3}+q^{1} .
$$

The Khovanov Homology of the link $\widehat{x_{1}^{3}}$ is presented in Table 2.

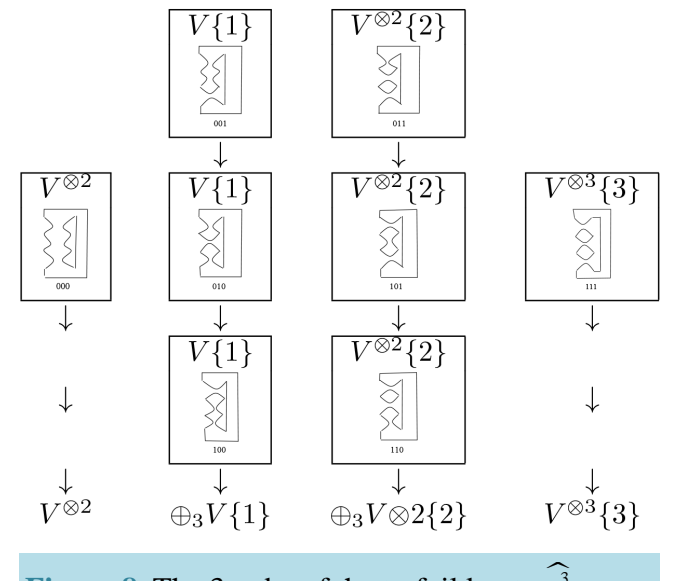

Figure 8. The 3-cube of the trefoil knot $\widehat{x_{1}^{3}}$. 
Table 1. Khovanov Brackets.

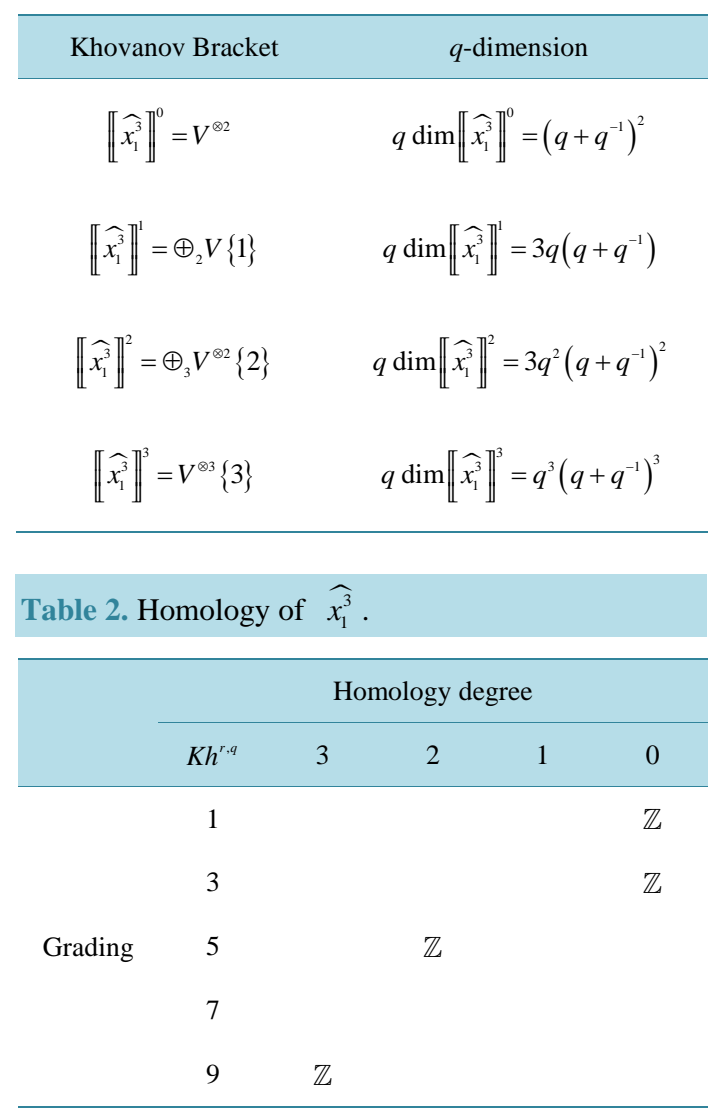

Remark 2. $\chi_{q}\left(\widehat{x_{1}^{3}}\right)$ is actually the unnormalized Jones polynomial of $\widehat{x_{1}^{3}}$.

\section{The Main Theorem}

This section contains the chain complex, Khovanov bracket, graded Euler characteristic, and Khovanov homology of the braid link $\widehat{x_{1}^{n}}$.

Proposition 4. The chain complex of the link $\widehat{x_{1}^{n}}$ is

$$
\left(\begin{array}{l}
n \\
0
\end{array}\right) V^{\otimes 2} \rightarrow\left(\begin{array}{l}
n \\
1
\end{array}\right) V \rightarrow\left(\begin{array}{l}
n \\
2
\end{array}\right) V^{\otimes 2} \rightarrow \cdots \rightarrow\left(\begin{array}{c}
n \\
n-1
\end{array}\right) V^{\otimes(n-1)} \rightarrow\left(\begin{array}{l}
n \\
n
\end{array}\right) V^{\otimes n} .
$$

Proof. We proof it by induction on $n$, using the trick that instead of “ $\rightarrow$ ”, we use “+” and that instead of $V^{\otimes 2}$, we use 1 just for first term in the expansion of $(1+V)^{\otimes n}$.

The expansion holds obviously for $n=1$, that is

$$
(1+V)^{1}=\left(\begin{array}{l}
1 \\
0
\end{array}\right) 1+\left(\begin{array}{l}
1 \\
1
\end{array}\right) V .
$$

Now, suppose that the result holds for $n=k$, that is

$$
(1+V)^{\otimes k}=\left(\begin{array}{l}
k \\
0
\end{array}\right) 1+\left(\begin{array}{l}
k \\
1
\end{array}\right) V+\cdots+\left(\begin{array}{c}
k \\
k-1
\end{array}\right) V^{\otimes(k-1)}+\left(\begin{array}{l}
k \\
k
\end{array}\right) V^{\otimes k}
$$

For $n=k+1$, we have 


$$
\begin{aligned}
(1+V)^{\otimes(k+1)} & =(1+V)(1+V)^{\otimes k} \\
& =\left[\left(\begin{array}{l}
1 \\
0
\end{array}\right) 1+\left(\begin{array}{l}
1 \\
1
\end{array}\right) V\right]\left[\left(\begin{array}{l}
k \\
0
\end{array}\right) 1+\left(\begin{array}{l}
k \\
1
\end{array}\right) V+\left(\begin{array}{l}
k \\
2
\end{array}\right) V^{\otimes 2}+\cdots+\left(\begin{array}{c}
k \\
k-1
\end{array}\right) V^{\otimes(k-1)}+\left(\begin{array}{l}
k \\
k
\end{array}\right) V^{\otimes k}\right] \\
& =\left(\begin{array}{c}
k \\
0
\end{array}\right) 1+\left(\left(\begin{array}{l}
k \\
1
\end{array}\right)+\left(\begin{array}{c}
k \\
0
\end{array}\right)\right) V+\cdots+\left(\left(\begin{array}{c}
k \\
k
\end{array}\right)+\left(\begin{array}{c}
k \\
k-1
\end{array}\right)\right) V^{\otimes k}+\left(\begin{array}{l}
k \\
k
\end{array}\right) V^{\otimes(k+1)} \\
& =1+\left(\begin{array}{c}
k+1 \\
1
\end{array}\right) V+\cdots+\left(\begin{array}{c}
k+1 \\
k
\end{array}\right) V^{\otimes k}+\left(\begin{array}{c}
k+1 \\
k+1
\end{array}\right) V^{\otimes(k+1)}
\end{aligned}
$$

Now, replacing 1 by $V^{\otimes 2}$ and “ $\rightarrow$ ” by “+”, we receive the desired result.

Theorem 5. The graded Euler characteristic of $\widehat{x_{1}^{n}}$ is

$$
\chi\left(\widehat{x_{1}^{n}}\right)=\left(q+q^{-1}\right)^{2}+\sum_{k=1}^{n}\left(\begin{array}{l}
n \\
k
\end{array}\right)(-q)^{k}\left(q+q^{-1}\right)^{k} .
$$

Proof. The proof is simple; just by following the definition.

Proposition 6. The unnormalized Jones polynomial of $x_{1}^{n}$ is

$$
\hat{J}\left(\widehat{x_{1}^{n}}\right)=(-1)^{n} q^{3 n}+q^{2}+q^{-2}+1
$$

and the normalized is

$$
J\left(\widehat{x_{1}^{n}}\right)=(-1)^{n} q^{3 n-1}+(-1)^{n-1} q^{3 n-3}+\cdots+(-1)^{2 n+1} q^{n+5}+q^{n+3}+q^{n-1} .
$$

Proof. Since the unnormalized Jones polynomial is the alternative sum of Khovanov brackets, we have

$$
\begin{aligned}
\hat{J}\left(\widehat{x_{1}^{n}}\right)= & \left(q+q^{-1}\right)^{2}-\left(\begin{array}{c}
n \\
1
\end{array}\right)\left[q\left(q+q^{-1}\right)\right]+\left(\begin{array}{l}
n \\
2
\end{array}\right)\left[q\left(q+q^{-1}\right)\right]^{2}-\left(\begin{array}{l}
n \\
3
\end{array}\right)\left[q\left(q+q^{-1}\right)\right]^{3} \\
& +\cdots+(-1)^{n-1}\left(\begin{array}{c}
n \\
n-1
\end{array}\right)\left[q\left(q+q^{-1}\right)\right]^{n-1}+(-1)^{n}\left(\begin{array}{l}
n \\
n
\end{array}\right)\left[q\left(q+q^{-1}\right)\right]^{n} \\
= & q^{2}+q^{-2}+2-\left(\begin{array}{l}
n \\
1
\end{array}\right)\left(q^{2}+1\right)+\left(\begin{array}{l}
n \\
2
\end{array}\right)\left(q^{4}+2 q^{2}+1\right)-\left(\begin{array}{l}
n \\
3
\end{array}\right)\left(q^{6}+3 q^{4}+3 q^{2}+1\right) \\
& +\cdots+(-1)^{n-1}\left(\begin{array}{c}
n \\
n-1
\end{array}\right)\left[q^{2(n-1)}+(n-1) q^{2(n-1)-2}+\frac{(n-1)(n-2)}{2 !} q^{2(n-1)-4}\right. \\
& \left.+\cdots+\frac{(n-1)(n-2)}{2 !} q^{4}+(n-1) q^{2}+1\right] \\
& +(-1)^{n}\left[q^{2 n}+n q^{2 n-2}+\frac{n(n-1)}{2 !} q^{2 n-4}+\cdots+\frac{n(n-1)}{2 !} q^{4}+n q^{2}+1\right] .
\end{aligned}
$$

Now after cancelation of terms, which behave differently for even and odd $n$, we receive the desired result.

For instance, see the cases for $n=5,6$ :

$$
\begin{aligned}
\hat{J}\left(\widehat{x_{1}^{5}}\right)= & \left(q+q^{-1}\right)-\left(\begin{array}{l}
5 \\
1
\end{array}\right)\left[q\left(q+q^{-1}\right)\right]+\left(\begin{array}{l}
5 \\
2
\end{array}\right)\left[q\left(q+q^{-1}\right)\right]^{2} \\
& -\left(\begin{array}{l}
5 \\
3
\end{array}\right)\left[q\left(q+q^{-1}\right)\right]^{3}+\left(\begin{array}{l}
5 \\
4
\end{array}\right)\left[q\left(q+q^{-1}\right)\right]^{4}-\left(\begin{array}{l}
5 \\
5
\end{array}\right)\left[q\left(q+q^{-1}\right)\right]^{5} \\
= & q^{2}+q^{-2}+2-5\left(q^{2}+1\right)+10\left(q^{4}+2 q^{2}+1\right)-10\left(q^{6}+3 q^{4}+3 q^{2}+1\right) \\
& +5\left(q^{8}+4 q^{6}+6 q^{4}+4 q^{2}+1\right)-\left(q^{10}+5 q^{8}+10 q^{6}+10 q^{4}+5 q^{2}+1\right) \\
= & -q^{15}+q^{2}+q^{-2}+1
\end{aligned}
$$




$$
\begin{aligned}
\hat{J}\left(\widehat{x_{1}^{6}}\right)= & \left(q+q^{-1}\right)-\left(\begin{array}{l}
6 \\
1
\end{array}\right)\left[q\left(q+q^{-1}\right)\right]+\left(\begin{array}{l}
6 \\
2
\end{array}\right)\left[q\left(q+q^{-1}\right)\right]^{2}-\left(\begin{array}{l}
6 \\
3
\end{array}\right)\left[q\left(q+q^{-1}\right)\right]^{3} \\
& +\left(\begin{array}{l}
6 \\
4
\end{array}\right)\left[q\left(q+q^{-1}\right)\right]^{4}-\left(\begin{array}{l}
6 \\
5
\end{array}\right)\left[q\left(q+q^{-1}\right)\right]^{5}+\left(\begin{array}{l}
6 \\
6
\end{array}\right)\left[q\left(q+q^{-1}\right)\right]^{6} \\
= & q^{2}+q^{-2}+2-6\left(q^{2}+1\right)+15\left(q^{4}+2 q^{2}+1\right)-20\left(q^{6}+3 q^{4}+3 q^{2}+1\right) \\
& +15\left(q^{8}+4 q^{6}+6 q^{4}+4 q^{2}+1\right)-6\left(q^{10}+5 q^{8}+10 q^{6}+10 q^{4}+5 q^{2}+1\right) \\
& +1\left(q^{12}+6 q^{10}+15 q^{8}+20 q^{6}+15 q^{4}+6 q^{2}+1\right) \\
= & q^{18}+q^{2}+q^{-2}+1
\end{aligned}
$$

Theorem 7. (Main theorem) a) If $n$ is even, then

b) If $n$ is odd, then

$$
K h^{k}\left(\widehat{x_{1}^{n}}\right)= \begin{cases}\mathbb{Z} \oplus \mathbb{Z} & \text { if } k=n, \\ 0 & \text { if } k=n-1 .\end{cases}
$$

$$
K h^{k}\left(\widehat{x_{1}^{n}}\right)= \begin{cases}\mathbb{Z} & \text { if } k=n \\ \mathbb{Z} & \text { if } k=n-1\end{cases}
$$

c) If $n \in \mathbb{Z}_{+}$, then

$$
K h^{k}\left(\widehat{x_{1}^{n}}\right)= \begin{cases}0 & \text { if } n-1<k \leq 1, \\ \mathbb{Z} \oplus \mathbb{Z} & \text { if } k=0 .\end{cases}
$$

Proof. We prove it using the relation

$$
\hat{J}\left(\widehat{x_{1}^{n}}\right) q^{n}=(-1)^{n} q^{3 n}+q^{n+2}+q^{n}+q^{n-2}
$$

and establishing a table with the help of the quantum and homological gradings. The homological grading $r$ appears in a row and quantum grading $q$ appears in a column. The homological gradings receive alternating signs, starting positive sign from 0 ; a term with negative sign appears at an odd $r$, while the positive sign appears at an even $r$. The powers of $q$ in the relation represent the quantum grading. Corresponding to each term in the relation, a $\mathbb{Z}$ space appears in the table at the $(q, r)^{\text {th }}$ position.

a) In case of even number of crossings we receive a 2-component link; hence, at $n^{\text {th }}$ homological grading, two $x_{1}^{n}$ spaces appear, one at quantum grading $3 n$ and one at quantum grading $(n+2)$. Please see Table 3 for the homology of $x_{1}^{n}$, where $n$ is even.

b) However, in odd number of crossing we always receive a knot; this confirms that at highest homological grading there exists a $\mathbb{Z}$ space against the quantum grading $3 n^{\text {th }}$. Moreover, at $(n+2)^{\text {th }}$ quantum grading one $\mathbb{Z}$ space should appear with positive coefficient in the Equation (4). Thus, a $\mathbb{Z}$ space actually appears at the position $(n+2, n-1)$. The homology of $x_{1}^{n}$, where $n$ is odd, is given in Table 4 .

c) Since at height 0 we receive the space $V \otimes V$, at $0^{\text {th }}$ homological level there exist two $\mathbb{Z}$ spaces, one at $(n-2)^{\text {th }}$ and one at $n^{\text {th }}$ quantum gradings. This completes the proof.

Now we give the graded Euler characteristic of the 3-strand braid $\alpha(n)=x_{1} x_{2} x_{1} x_{2} \cdots$ (n factors); this sequence contains the powers of Garside element $\Delta_{3}=\Delta: \quad \alpha(3 k)=\Delta^{k}$. We will use Table 5 , where $X$ is the canonical form of $\alpha(n)$ (i.e. the smallest word in the length-lexicographic order with $x_{1}<x_{2}$ ) and $Y$ is a conjugate of $X$, suitable for computations. The number of factors in each of the six $Y$ is $2 k+2$.

\section{Theorem 8.}
1) $\chi_{q}\left(\Delta^{2 k}\right)=2 q^{7}+2 q^{5}+q^{3}+q+q^{-1}+q^{-3}$
2) $\chi_{q}\left(\Delta^{2 k} x_{1}\right)=-q^{9}+q^{5}+q^{3}+q+q^{-1}+q^{-3}$
3) $\chi_{q}\left(\Delta^{2 k} x_{1} x_{2}\right)=-q^{9}-q^{7}-q^{3}+q+q^{-1}+q^{-3}$
4) $\chi_{q}\left(\Delta^{2 k+1}\right)=q^{3}+q+q^{-1}+q^{-3}$ 
Table 3. Homology of $x_{1}^{n}$, where $n$ is even.

\begin{tabular}{ccccccccc}
\hline & $(-1)^{n}$ & $(-1)^{n-1}$ & $(-1)^{n-2}$ & $\cdots$ & - & + & - & + \\
\hline$K h^{q, r}$ & $n$ & $n-1$ & $n-2$ & $\cdots$ & 3 & 2 & 1 & 0 \\
$n-2$ & & & & & & & & $\mathbb{Z}$ \\
$n$ & & & & & & & \\
$n+2$ & $\mathbb{Z}$ & & & & & \\
$\vdots$ & & & & & & \\
$3 n$ & $\mathbb{Z}$ & & & & & \\
\hline
\end{tabular}

Table 4. Homology of $x_{1}^{n}$, where $n$ is odd.

\begin{tabular}{ccccccccc} 
& $(-1)^{n}$ & $(-1)^{n-1}$ & $(-1)^{n-2}$ & $\cdots$ & - & + & - & + \\
\hline$K h^{q, r}$ & $n$ & $n-1$ & $n-2$ & $\cdots$ & 3 & 2 & 1 & 0 \\
$n-2$ & & & & & & & & $\mathbb{Z}$ \\
$n$ & & & & & & & & $\mathbb{Z}$ \\
$n+2$ & & $\mathbb{Z}$ & & & & & & \\
$\vdots$ & & & & & & & \\
$3 n$ & $\mathbb{Z}$ & & & & & & \\
\hline
\end{tabular}

Table 5. Classification of the braid $\alpha(n)=x_{1} x_{2} x_{1} x_{2} \cdots$.

\begin{tabular}{ccc}
\hline$\alpha(n)$ & $X$ & $Y$ \\
\hline$\Delta^{2 k}$ & $x_{1}^{2 k} x_{2} x_{1}^{2} x_{2}^{2} \cdots x_{1}^{2} x_{2}$ & $x_{1}^{2 k} x_{2} x_{1}^{2} x_{2}^{2} \cdots x_{1}^{2} x_{2}$ \\
$\Delta^{2 k} x_{1}$ & $x_{1}^{2 k+1} x_{2} x_{1}^{2} x_{2}^{2} \cdots x_{1}^{2} x_{2}$ & $x_{1}^{2 k+1} x_{2} x_{1}^{2} x_{2}^{2} \cdots x_{1}^{2} x_{2}$ \\
$\Delta^{2 k} x_{1} X_{2}$ & $x_{1}^{2 k+1} x_{2} x_{1}^{2} x_{2}^{2} \cdots x_{1}^{2} x_{2}^{2}$ & $x_{1}^{2 k+1} x_{2} x_{1}^{2} x_{2}^{2} \cdots x_{1}^{2} x_{2}^{2}$ \\
$\Delta^{2 k+1}$ & $x_{1}^{2 k+1} x_{2} x_{1}^{2} x_{2}^{2} \cdots x_{2}^{2} x_{1}$ & $x_{1}^{2 k+2} x_{2} x_{1}^{2} x_{2}^{2} \cdots x_{1}^{2} x_{2}^{2}$ \\
$\Delta^{2 k+1} x_{2}$ & $x_{1}^{2 k+2} x_{2} x_{1}^{2} x_{2}^{2} \cdots x_{2}^{2} x_{1}$ & $x_{1}^{2 k+3} x_{2} x_{1}^{2} x_{2}^{2} \cdots x_{1}^{2} x_{2}^{2}$ \\
$\Delta^{2 k+1} x_{2} X_{1}$ & $x_{1}^{2 k+2} x_{2} x_{1}^{2} x_{2}^{2} \cdots x_{2}^{2} x_{1}^{2}$ & $x_{1}^{2 k+4} x_{2} x_{1}^{2} x_{2}^{2} \cdots x_{1}^{2} x_{2}^{2}$ \\
\hline
\end{tabular}

5) $\chi_{q}\left(\Delta^{2 k+1} x_{2}\right)=-q^{11}-q^{9}+q^{3}+q+q^{-1}+q^{-3}$

6) $\chi_{q}\left(\Delta^{2 k+1} x_{2} x_{1}\right)=q^{13}+q^{11}-q^{7}+q+q^{-1}+q^{-3}$

Proof. (4) Since there are $6 k+3$ crossings in the link $\Delta^{2 k+1}$, there are $2^{6 k+3}$ vertices in the smoothing cube. The Khovanov brackets along with their $q$-dimensions are given in Table 6.

The result now follows using the definition and simplifying the expression.

See, for example, the case for $k=1$. The figure on the right represent the link of the reduced form of $\Delta^{3}$,

which is $x_{1}^{4} x_{2} x_{1}^{2} x_{2}^{2}$.

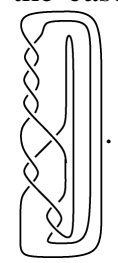


Table 6. Khovanov brackets and $q$-dimensions for smoothings of $\Delta^{2 k+1}$.

\begin{tabular}{ccc}
\hline Level & Khovanov Bracket & $q$-dimension \\
0 & $V^{\otimes 3}$ & $\left(q+q^{-1}\right)^{3}$ \\
1 & $\oplus_{9} V^{\otimes 2}\{1\}$ & $(6 k+3) q\left(q+q^{-1}\right)^{2}$ \\
2 & $\oplus_{18} V^{\otimes 1}\{2\} \oplus_{18} V^{\otimes 3}\{2\}$ & $q^{2}\left[\left(\begin{array}{c}2 k+1 \\
1\end{array}\right)\left(\begin{array}{c}4 k+2 \\
1\end{array}\right)\left(q+q^{-1}\right)+\left(\left(\begin{array}{c}2 k+1 \\
2\end{array}\right)+\left(\begin{array}{c}4 k+2 \\
2\end{array}\right)\right)\left(q+q^{-1}\right)^{3}\right]$ \\
3 & $\oplus_{63} V^{\otimes 2}\{3\} \oplus_{21} V^{\otimes 4}\{3\}$ & $q^{3}\left[\left(\begin{array}{c}2 k+1 \\
1\end{array}\right)\left(\begin{array}{c}4 k+2 \\
2\end{array}\right)+\left(\begin{array}{c}2 k+1 \\
2\end{array}\right)\left(\begin{array}{c}4 k+2 \\
1\end{array}\right)\left(q+q^{-1}\right)^{2}+\left(\left(\begin{array}{c}2 k+1 \\
3\end{array}\right)+\left(\begin{array}{c}4 k+2 \\
3\end{array}\right)\right)\left(q+q^{-1}\right)^{4}\right]$ \\
$\vdots$ & $\vdots$ \\
$6 k+2$ & $(2 k+1) V^{\otimes 2 k+3}+(4 k+2) V^{\otimes 2 k+1}$ & $q^{6 k+2}\left[(2 k+1)\left(q+q^{-1}\right)^{2 k+3}+(4 k+2)\left(q+q^{-1}\right)^{2 k+1}\right]$ \\
$6 k+3$ & $V^{8 k+3}\left(q+q^{-1}\right)^{2 k+2}$
\end{tabular}

Table 7. Khovanov bracket and $q$-dimensions for smoothings of $\Delta^{3}$.

\begin{tabular}{ccc}
\hline Level & Khovanov bracket & $q$-dimension \\
\hline 0 & $V^{\otimes 3}$ & $\left(q+q^{-1}\right)^{3}$ \\
1 & $\oplus_{9} V^{\otimes 2}\{1\}$ & $9 q\left(q+q^{-1}\right)^{2}$ \\
2 & $\oplus_{18} V^{\otimes 1}\{2\} \oplus_{18} V^{\otimes 3}\{2\}$ & $18 q^{2}\left(q+q^{-1}\right)+18 q^{2}\left(q+q^{-1}\right)^{3}$ \\
3 & $\oplus_{63} V^{\otimes 2}\{3\} \oplus_{21} V^{\otimes 4}\{3\}$ & $63 q^{3}\left(q+q^{-1}\right)^{2}+4 q^{21}\left(q+q^{-1}\right)^{4}$ \\
4 & $\oplus_{24} V^{\otimes 1}\{4\} \oplus_{87} V^{\otimes 3}\{4\} \oplus_{15} V^{\otimes 5}\{4\}$ & $24 q^{4}\left(q+q^{-1}\right)+87 q^{4}\left(q+q^{-1}\right)^{3}+15 q^{4}\left(q+q^{-1}\right)^{5}$ \\
5 & $\oplus_{60} V^{\otimes 2}\{5\} \oplus_{60} V^{\otimes 4}\{5\} \oplus_{6} V^{\otimes 6}\{5\}$ & $60 q^{5}\left(q+q^{-1}\right)^{2}+60 q^{5}\left(q+q^{-1}\right)^{4}+6 q^{5}\left(q+q^{-1}\right)^{6}$ \\
7 & $\oplus_{8} V^{\otimes 1}\{6\} \oplus_{54} V^{\otimes 3}\{6\} \oplus_{21} V^{\otimes 5}\{6\} \oplus_{1} V^{\otimes 7}\{6\}$ & $8 q^{6}\left(q+q^{-1}\right)^{1}+54 q^{6}\left(q+q^{-1}\right)^{3}+21 q^{6}\left(q+q^{-1}\right)^{5}+q^{6}\left(q+q^{-1}\right)^{7}$ \\
8 & $\oplus_{12} V^{\otimes 2}\{7\} \oplus_{21} V^{\otimes 4}\{7\} \oplus_{3} V^{\otimes 6}\{7\}$ & $12 q^{7}\left(q+q^{-1}\right)^{2}+21 q^{7}\left(q+q^{-1}\right)^{4}+3 q^{7}\left(q+q^{-1}\right)^{6}$ \\
9 & $\oplus_{6} V^{\otimes 3}\{8\} \oplus_{3} V^{\otimes 5}\{7\}$ & $6 q^{8}\left(q+q^{-1}\right)^{3}+3 q^{8}\left(q+q^{-1}\right)^{5}$ \\
\end{tabular}

Table 8. Homology of $\Delta^{3}$.

\begin{tabular}{|c|c|c|c|c|c|c|c|c|c|c|c|}
\hline \multicolumn{12}{|c|}{ Homological grading $q$} \\
\hline & $K h^{r, q}$ & 9 & 8 & 7 & 6 & 5 & 4 & 3 & 2 & 1 & 0 \\
\hline \multirow{4}{*}{$\begin{array}{c}\text { Quantum } \\
\text { grading } \\
r\end{array}$} & 6 & & & & & & & & & & $\mathbb{Z}$ \\
\hline & 8 & & & & & & & & & & $\mathbb{Z}$ \\
\hline & 10 & & & & & & & & $\mathbb{Z}$ & & \\
\hline & 12 & & & & & & & & $\mathbb{Z}$ & & \\
\hline
\end{tabular}


For Khovanov brackets and q-dimensions for smoothings of $\Delta^{3}$ (see Table 7). We ultimately receive $\chi_{q}\left(\Delta^{3}\right) q^{9}=q^{12}+q^{9}+q^{8}+q^{6}$. The homology of $\Delta^{3}$ is presented in Table 8 .

The proofs of other parts are similar to the proof of Part 4.

\section{References}

[1] Khovanov, M. (2000) A Categorification of the Jones Polynomial. Duke Mathematical Journal, 3, 359-426.

[2] Bar-Natan, D. (2002) On Khovanov's Categorification of the Jones Polynomial. Algebraic and Geometric Topology, 2, 337-370. http://dx.doi.org/10.2140/agt.2002.2.337

[3] Reidemeister, K. (1926) Elementare begrundung der knotentheorie. Abhandlungen aus dem Mathematischen Seminar der Universität Hamburg, 5, 24-32.

[4] Manturov, V. (2004) Knot Theory. Chapman and Hall/CRC, Boca Raton.

[5] Artin, E. (1947) Theory of Braids. Annals of Mathematics, 48,101-126. http://dx.doi.org/10.2307/1969218

[6] Alexander, J. (1923) Topological Invariants of Knots and Links. Transactions of the American Mathematical Society, 20, 275-306.

[7] Jones, V. (1985) A Polynomial Invariant for Knots via Von Neumann Algebras. Bulletin of the American Mathematical Society, 12, 103-111. http://dx.doi.org/10.1090/S0273-0979-1985-15304-2

[8] Kauffman, L.H. (1987) State Models and the Jones Polynomial. Topology, 26, 395-407. http://dx.doi.org/10.1016/0040-9383(87)90009-7

[9] Reidemeister, K. (1948) Knot Theory. Chelsea Publ. and Co., New York. 